Research Paper

\title{
CT features and outcomes of newly developed pulmonary lesions in patients with Coronavirus Disease 2019 (COVID-19)
}

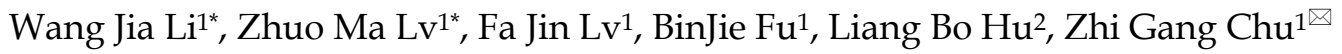 \\ 1. Department of Radiology, The First Affiliated Hospital of Chongqing Medical University, Chongqing, China, 40016. \\ 2. Department of Radiology, Yongchuan Hospital of Chongqing Medical University, Chongqing, China, 40016. \\ ${ }^{*}$ Co-first authors with equal contributions to this work.
}

$\triangle$ Corresponding author: Zhigang Chu, MD, PhD. Department of Radiology, The First Affiliated Hospital of Chongqing Medical University, 1\# Youyi Road, Yuanjiagang, Yuzhong district, Chongqing, China, 400016. E-mail: chuzg0815@163.com; Tel.: +86 18723032809; Fax: +86 23 68811487.

(C) The author(s). This is an open access article distributed under the terms of the Creative Commons Attribution License (https://creativecommons.org/licenses/by/4.0/). See http://ivyspring.com/terms for full terms and conditions.

Received: 2020.04.29; Accepted: 2020.08.15; Published: 2020.08.29

\begin{abstract}
Background: In patients with coronavirus disease 2019 (COVID-19) pneumonia, whether new pulmonary lesions will continue to develop after treatment was unknown. This study aimed to determine whether new pulmonary lesions will develop after treatment in patients with COVID-19 pneumonia, and investigate their CT features and outcomes.

Methods: This retrospective study included 56 consecutive patients with confirmed COVID-19 pneumonia from January 20 to March 5, 2020. Their initial and follow-up CT images and clinical data were reviewed. The CT manifestations of primary and newly developed pulmonary lesions and their changes after treatment were mainly evaluated.

Results: Among the 56 patients (mean age: $48 \pm 15$ years, 35 men) with COVID-19 pneumonia, 42 (75.0\%) patients developed new pulmonary lesions during treatment. All new lesions developed before the nucleic acid test turned negative. Patients with new lesions were more likely to have lymphopenia $(P=0.041)$ or increased C-reactive protein $(C R P)$ levels $(P<0.001)$ than those without new lesions. Of the 42 patients, $30(71.4 \%)$ patients developed new lesions once, and 12 (28.6\%) twice or thrice, which usually appeared when primary lesions were progressing $(37,88.1 \%)$ and $1-15$ days after treatment. The newly developed lesions were usually multiple $(38,90.5 \%)$, distributed in the previously involved $(39,92.9 \%)$ or uninvolved $(27,64.3 \%)$ lobes, and manifested as ground-glass opacities (GGOs) with consolidation $(23,54.8 \%)$ or pure GGOs $(19,45.2 \%)$. After their occurrence, the new lesions in most patients (32,76.2\%) showed direct absorption, whereas those in some patients (10,23.8\%) progressed before absorption.

Conclusion: During treatment, most patients with COVID-19 pneumonia will develop new pulmonary lesions, which usually manifest as multiple GGOs distributed around the primary lesions or in previously uninvolved lobes, and are subsequently absorbed directly.
\end{abstract}

Key words: Coronavirus Disease 2019, Pneumonia, New lesions, Primary lesions, CT

\section{Introduction}

Coronavirus disease 2019 (COVID-19) is a global pandemic caused by severe acute respiratory syndrome coronavirus 2 (SARS-CoV-2) [1, 2]. Although SARS-CoV-2 has at least $70 \%$ similarity in genetic sequence to SARS-CoV [3], COVID-19 spreads much more rapidly than SARS [4]. This might be because the initial symptoms of patients with COVID-19 pneumonia are not obvious and nonspecific, and asymptomatic carriers can transmit SARS-CoV-2 to other uninfected people, causing the virus to spread rapidly.

SARS-CoV-2 can be transmitted to others through droplets from infected patients [5]. Also, the virus could be transmitted from primary lesions to the infected or uninfected lung tissue in patients with COVID-19 pneumonia. COVID-19 testing is 
performed by using nucleic acid tests of SARS-CoV-2, which remains the reference standard for diagnosing the disease [6]. Multiple viral nucleic acid tests are used to determine whether patients still carry the virus and are contagious after treatment. However, it is unclear whether these tests can evaluate the patients' self-transmission accurately.

Since nucleic acid test can result in false-negative outcomes, chest CT plays a vital role in early diagnosis and follow-up of COVID-19 [7, 8]. The imaging characteristics and evolution rules of pulmonary lesions revealed in previous studies provided useful information for understanding this disease [9-13]. However, they did not mention the newly developed lesions and analyze their CT features and outcomes. Therefore, this study aimed to determine whether patients with COVID-19 pneumonia would develop new lesions after treatment, and their related clinical factors, and to investigate their CT characteristics and following changes for understanding self-transmission better.

\section{Materials and Methods}

This retrospective study used unidentifiable patient data and had no potential risk to patients. Therefore, the institutional review board of our hospital approved this study and waived informed consent.

\section{Study population}

From January 20 to March 5, 2020, patients with confirmed COVID-19 were enrolled in this study. Upon admission, all patients underwent chest CT examinations. Follow-up CT scans were obtained during treatment. Two patients without complete clinical data, 13 patients without pulmonary lesions on initial $\mathrm{CT}$, and six patients with the interval between two consecutive CT scans of $>7$ days were excluded from the study. The flowchart of study population is shown in Figure 1.

\section{CT examinations}

All patients underwent CT examinations upon admission to our hospital. Repeat follow-up CT scans for each patient were performed. All CT examinations were performed using two multi-detector $\mathrm{CT}$ scanners (Philips Brilliance iCT; Philips Healthcare, Massachusetts, United States, and Siemens SOMATOM go.Top; Siemens Healthineers, Erlangen, Germany) with the following parameters: $120 \mathrm{kV}$, automatic tube current modulation; detector collimation, $0.625 / 0.6 \mathrm{~mm}$; rotation time, $0.5 \mathrm{~s}$; pitch, 0.758/1.5; matrix: $512 \times 512$; section thickness and interval, 5.0 and $5.0 \mathrm{~mm}$, respectively; and reconstructed thickness, $1 \mathrm{~mm}$. All non-contrast CT scans were acquired with patients in a supine position and the patients were scanned from the thoracic inlet to the lung base at the end of inspiration.

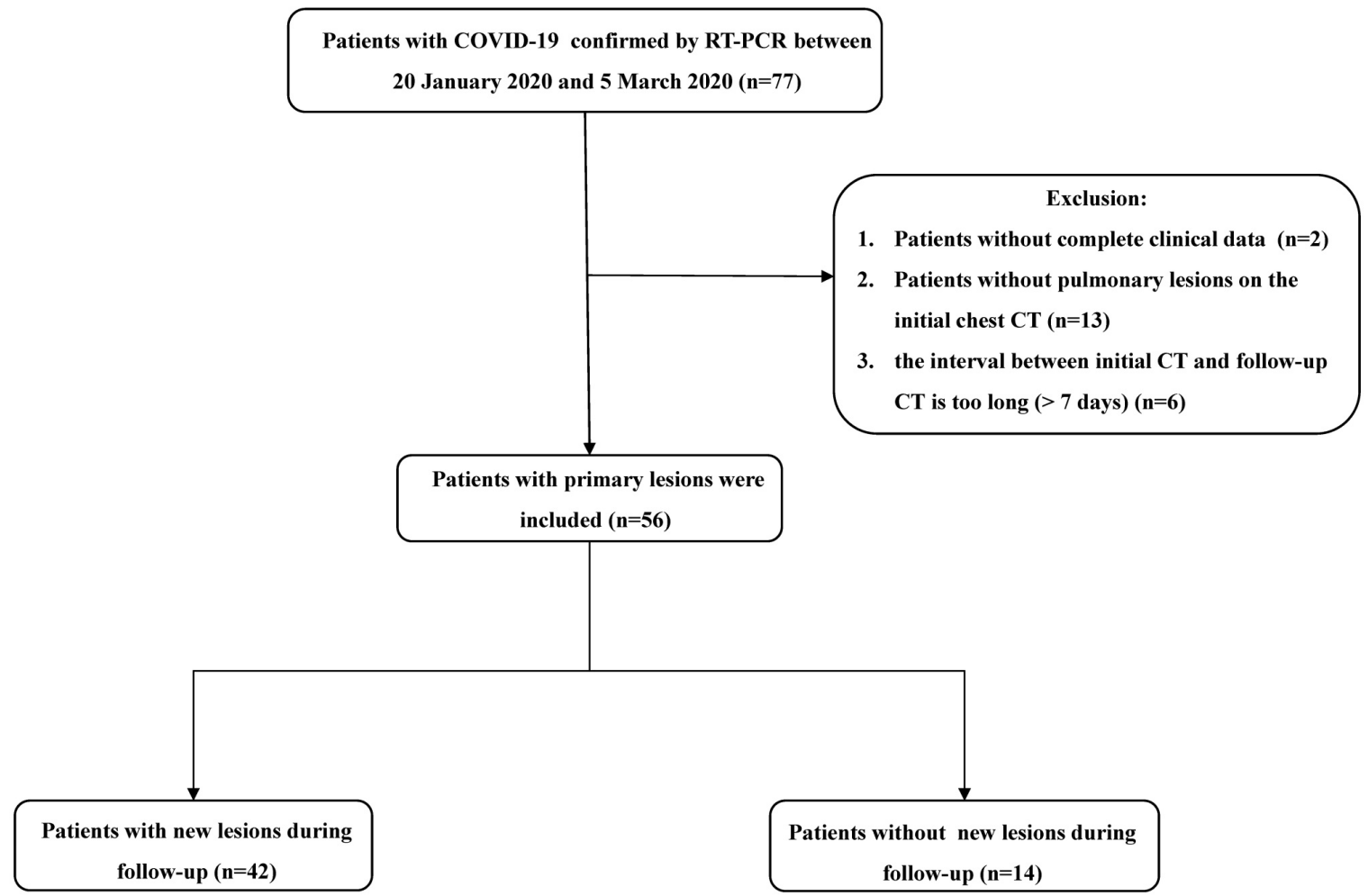

Figure 1. Flowchart of study population. Abbreviations: COVID-19: coronavirus disease 2019; GGOs: ground-glass opacities. 


\section{Analysis of CT characteristics}

All CT data were analyzed on the picture archiving and communication system with a lung window setting (width, $1500 \mathrm{HU}$; level, $-500 \mathrm{HU}$ ). Two senior radiologists (Z.G.C and F.J.L with 10 and 20 years of experience in thoracic imaging, respectively), who were blinded to the clinical data, independently evaluated the initial and follow-up CT images. The discrepancies were resolved by consensus.

For each patient, the distribution, extent, and CT features of the primary and newly developed lesions were evaluated. On follow-up CT images, changes in the primary and new lesions were evaluated. The new lesions were evaluated in the following aspects: (a) interval of initial CT to the first repeat CT with newly developed lesions, (b) number (single or multiple); (c) distribution (in previously involved lobes, in previously uninvolved lobes, or both); (d) density (pure GGO, GGOs with consolidation, or both), (e) shape (small patchy, nodular, or both), and (f) their changes during treatment (directly absorption or progression before absorption). Based on the presence or absence of newly developed lesions during treatment, the patients were divided into two groups: cases with new lesions and cases without new lesions.

\section{Clinical characteristics}

Each patients' clinical and laboratory data were recorded by one radiologist (W.J.L). The clinical data included age, gender, clinical type, and initial symptoms. Laboratory findings, such as white blood cell, lymphocyte, C-reactive protein (CRP), lactate dehydrogenase (LDH), and partial pressure of oxygen $\left(\mathrm{PaO}_{2}\right)$, were recorded. These laboratory tests were performed when the patients admitted to the hospital. Also, the frequency and results of nucleic acid tests were recorded.

\section{Statistical analysis}

All data were statistically analyzed using Statistical Packages for the Social Sciences, version 20.0 (IBM Corp., Armonk, New York, USA). Data were expressed as mean \pm standard deviation for continuous variables, and presented as numbers and percentages for categorical variables. The analysis of variance or Wilcoxon rank-sum test was used for continuous variables, and categorical variables were analyzed using the Pearson $\chi^{2}$ test or Fisher exact test. A $\mathrm{P}$ value of $\leq 0.05$ was considered statistically significant.

\section{Results}

Fifty-six patients, including $35(62.5 \%)$ men (mean age, $48 \pm 15$ years; range, $14-89$ years) and 21 (37.5\%) women (mean age, $52 \pm 13$ years; range, 26-83 years), with a mean age of $48 \pm 15$ years (range, 14-89 years), were enrolled in this study. Of the 56 patients, $42(75.0 \%)$ developed new lesions during treatment. The patients' clinical and laboratory data are shown in Table 1. The mean duration from illness onset to admission was three days. Fever (38/56, 67.9\%) and cough (38/56, 67.9\%) were the most common symptoms. More patients with new lesions showed lymphopenia and increased CRP than those without $(P=0.041$ and $P<0.001$, respectively). All patients were confirmed to have a positive result for COVID-19 from nucleic acid tests of respiratory secretions upon admission and turned negative (twice in succession) before discharged. Besides, the nucleic acid tests of SARS-CoV-2 in all patients with new lesions turned negative after all new lesions developed.

\section{CT imaging findings}

Of the 56 patients, primary pulmonary lesions were usually multiple $(53,94.6 \%)$, frequently involved multiple lobes $(38,67.9 \%)$, mainly distributed in the lower lobes $(47,83.9 \%)$, and manifested as pure GGOs $(33,58.9 \%)$ and GGOs with consolidation $(23,41.1 \%)$. The involved lobes ( $3.5 \pm 1.5$ vs. $3.1 \pm 1.4, P=0.402)$ and segments $(7.9 \pm 5.0$ vs. $7.2 \pm 1.4, P=0.489)$ in patients with and without new lesions were similar.

Table 2 presents the CT features of newly developed pulmonary lesions in patients with COVID-19. Among 42 patients with new lesions, 23 $(54.8 \%)$ developed new lesions $1-4$ days after the initial CT. New lesions developed once in $30(71.4 \%)$ cases, and twice and three times in $12(28.6 \%)$ cases. They were usually multiple (38/42, 90.5\%), distributed around primary lesions $(39 / 42,92.9 \%)$ or in the previously uninvolved lobes $(27 / 42,64.3 \%)$, and manifested as GGOs with consolidation (23/42, $54.8 \%)$ or pure GGOs $(19 / 42,45.2 \%)$. The number of new lesions developed in different periods was similar $(P=0.377)$, with a mean number of $6.2 \pm 4.8$.

\section{Changes of primary and newly developed lesions during follow-up}

Table 3 presents follow-up CT characteristics of patients with COVID-19. During treatment, primary lesion progression was significantly more common in patients with new lesions than in those without. (90.5\% vs. $14.3 \%, P=0.000)$. Most patients $(37 / 42$, $88.1 \%)$ developed new lesions when the primary lesions progressed. After their occurrence, most of the newly developed lesions (32, 76.2\%) were directly absorbed (Figure 2). On the latest CT, patients with new lesions had more complex residues than those without new lesions. 


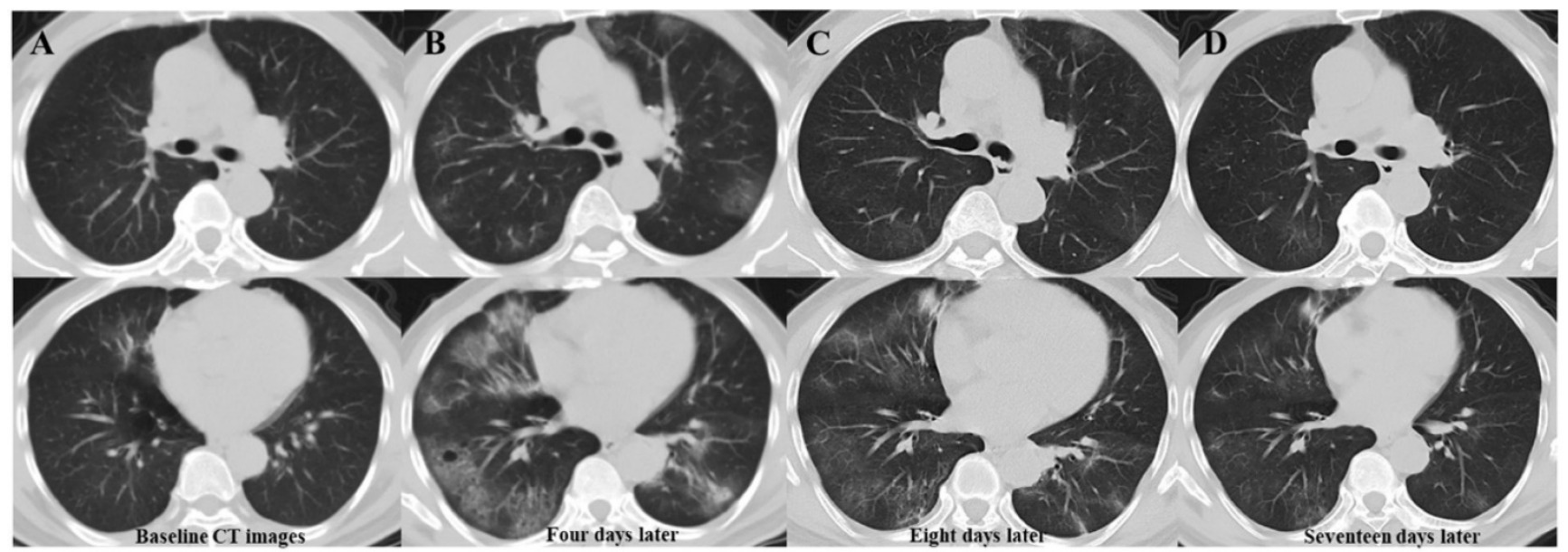

Figure 2. Serial chest CT images of a 63-year-old man with confirmed COVID-19 pneumonia. Baseline CT images (column A) show patchy ground-glass opacity (GGO) in the right middle lobe. Four days later (column B), the primary lesion progress, and multiple new GGOs occur in previously involved and uninvolved lobes. Follow-up CT images (column C) eight days after admission show absorption of the primary and newly developed lesions. The latest follow-up CT images (column D) 17 days after admission show significant absorption of lesions, with little residue. Abbreviations: COVID-19: coronavirus disease 2019; GGOs: ground-glass opacities.

Table 1. The clinical and laboratory data of patients with COVID-19

\begin{tabular}{|c|c|c|c|}
\hline & $\begin{array}{l}\text { Patients with new } \\
\text { lesions }(n=42)\end{array}$ & $\begin{array}{l}\text { Patients without } \\
\text { new lesions }(n=14)\end{array}$ & $P$-value \\
\hline Age & $49.6 \pm 16.1(14-89)$ & $44.1 \pm 13.0(16-67)$ & $0.253 \&$ \\
\hline \multicolumn{4}{|l|}{ Gender } \\
\hline Man & $28(66.7)$ & $8(57.0)$ & $0.520^{*}$ \\
\hline Woman & $14(33.3)$ & $6(43.0)$ & \\
\hline \multicolumn{4}{|l|}{ Clinical type } \\
\hline Mild & $2(4.8)$ & $0(0)$ & \\
\hline Moderate & $34(81.0)$ & $13(93.0)$ & $0.811^{\text {** }}$ \\
\hline Severe & $6(14.3)$ & $1(7.0)$ & \\
\hline \multicolumn{4}{|c|}{ Clinical manifestations at admission } \\
\hline Fever & $30(71.4)$ & $8(57.0)$ & \\
\hline Cough & $28(66.7)$ & $10(71.0)$ & \\
\hline Sputum & $8(19.0)$ & $3(21.0)$ & \\
\hline Dyspnea & $4(9.5)$ & $3(21.0)$ & \\
\hline Diarrhea & $4(9.5)$ & $2(14.0)$ & \\
\hline \multicolumn{4}{|l|}{ Laboratory indicators } \\
\hline leucocyte $\left(\times 10^{9} / \mathrm{L}, 3.5-9.5\right)$ & $4.9 \pm 2.1(1.5-11.8)$ & $5.9 \pm 1.8(2.0-8.9)$ & \\
\hline Decreased & $8(19.0)$ & $1(7.0)$ & \\
\hline Normal & $32(76.2)$ & $13(93.0)$ & $0.376^{* *}$ \\
\hline Increased & $2(4.8)$ & $0(0)$ & \\
\hline $\begin{array}{l}\text { Lymphocyte } \\
\left(\times 10^{9} / \mathrm{L}, 1.1-3.2\right)\end{array}$ & $1.0 \pm 0.5(0.4-2.8)$ & $1.2 \pm 0.5(0.2-2.1)$ & \\
\hline Decreased & $28(66.7)$ & $5(36.0)$ & $0.041^{*}$ \\
\hline Normal & $14(33.3)$ & $9(64.0)$ & \\
\hline CRP (mg/L, 0-8) & $25.6 \pm 24.6(0.5-94.9)$ & $14.2 \pm 33.2(0.5-124.1)$ & \\
\hline Increased & $33(78.6)$ & $2(14.0)$ & $0.000^{*}$ \\
\hline Normal & $9(21.4)$ & $12(86.0)$ & \\
\hline LDH (U/L, 12-250) & $535 \pm 173(142-1024)$ & $440 \pm 166(177-860)$ & \\
\hline Increased & $39(92.9)$ & $13(93.0)$ & $1.000^{*}$ \\
\hline Normal & $3(7.1)$ & $1(7.0)$ & \\
\hline $\mathrm{PaO} 2$ (kpa, 80-100) & $91 \pm 28(58-179)$ & $109 \pm 37(54-167)$ & \\
\hline Decreased & $15(35.7)$ & $4(28.6)$ & \\
\hline Normal & $17(40.5)$ & $4(28.6)$ & $0.553^{\text {** }}$ \\
\hline Increased & $10(23.8)$ & $6(42.8)$ & \\
\hline Follow-up time (d) & $18 \pm 5(10-31)$ & $15 \pm 5(10-29)$ & $0.128^{*}$ \\
\hline Numbers of CT scans & $4 \pm 1(2-6)$ & $4 \pm 1(2-6)$ & $0.294^{\#}$ \\
\hline
\end{tabular}

Notes: (a) Continuous data were presented as mean \pm standard deviation (minimum-maximum), while the categorical data were presented as count (percentage of the total); (b) Abbreviations: COVID-19: coronavirus disease 2019; CRP: C-reactive protein; $\mathrm{LDH}$ : lactate dehydrogenase; $\mathrm{PaO}_{2}$ : partial pressure of oxygen; (c) *Pearson $\chi^{2}$ test; ${ }^{* *}$ Fisher exact test; \# Wilcoxon rank sum test; \& Analysis of variance.

\section{Discussion}

The present study revealed that the development of new pulmonary lesions during treatment was common, and was mainly related to lymphopenia, elevated CRP, or primary lesions progression, but irrelevant to the extent of primary lesions and other clinical characteristics. Previous studies have revealed that COVID-19 may be associated with cellular immune and acute severe systemic inflammatory responses [14, 15]. Therefore, the development of new lesions indicated that primary lesions were more severe and could not be well-controlled by conventional treatment. Also, more complex residues on the latest $\mathrm{CT}$ in patients with progressed primary lesions also confirmed this finding. It might be because the population is susceptible to this disease, and there is no specific treatment at present, so patients mainly rely on autoimmunity [16, 17]. Thus, the patients with worsening laboratory indicators should be monitored closely.

In this study, new lesions developed in the early stage of the disease, indicating that COVID-19 cannot be controlled quickly, and SARS-CoV-2 can be transmitted easily in the early stage, which was consistent with other results $[4,14,18]$. The SARS-CoV-2 load usually peaked at the symptom onset, which was different from that of SARS-CoV and MERS-CoV $[19,20]$. The high viral load of SARS-CoV-2 in the early stage represents its high infectivity in this period, which could explain its extensive transmission. Over time, the viral load gradually declines, indicating that the infectivity gradually decreases during treatment. However, some patients in this study still developed new lesions up to 15 days after treatment. Thus, the disease's longer communicable period should be given enough 
attention. For patients with new lesions, their nucleic acid test turned negative after all new lesions appeared, which showed a good consistency between $\mathrm{CT}$ and nucleic acid test results. Therefore, these two diagnostic methods should be combined to determine the potential infectivity from macroscopic and microscopic views.

Table 2. The CT features of new lesions in patients with COVID-19

\begin{tabular}{ll}
\hline Characteristics & \multicolumn{1}{c}{ Patients with new lesions $(\mathrm{n}=42)$} \\
\hline Interval of initial CT to the first repeat CT with new lesions \\
1-4 day & $23(54.8)$ \\
5-8 day & $8(19.0)$ \\
$9-12$ day & $8(19.0)$ \\
13-15 day & $3(7.1)$ \\
Number & \\
Multiple & $38(90.5)$ \\
Solidary & $4(9.5)$ \\
1-4 day & $6.3 \pm 4.8(1-20)$ \\
5-8 day & $7.6 \pm 5.8(1-18)$ \\
9-12 day & $6.1 \pm 4.8(1-15)$ \\
13-15day & $3.2 \pm 3.2(1-9)$ \\
Distribution & \\
Previously involved lobes & $15(35.7)$ \\
Previously uninvolved lobes & $3(7.1)$ \\
Both & $24(57.1)$ \\
Density & \\
Pure GGOs & $19(45.2)$ \\
GGOs with consolidation & $23(54.8)$ \\
Shape & $39(92.9)$ \\
Small patchy & $14(33.3)$ \\
Nodular & $11(26.2)$ \\
Both &
\end{tabular}

Note: Continuous data were presented as mean \pm standard deviation (minimummaximum), while the categorical data were presented as count (percentage of the total);

Abbreviations: COVID-19: coronavirus disease 2019, GGOs: ground-glass opacities.

On CT images, newly developed lesions at any period were usually multiple and distributed not only in previously involved lobes but also in uninvolved lobes, which was consistent with SARS-CoV-2's strong infectivity. Thus, the rapid progression of pulmonary lesions attributed to both the development and dissemination of lesions. GGOs with or without consolidation were the main CT manifestations of COVID-19 pneumonia, which was also found in other studies [21-23]. Regarding new lesions, they showed similar manifestations with primary lesions but had a relatively limited extent. Most of them were absorbed directly after occurrence, which suggested that the treatment efficiently decreased the virus' ability to damage lung tissue and controlled its progression.

This study has two limitations. First, the interval and number of follow-up CT scans in different patients were different, so the occurrence time of new lesions may be inaccurate. However, multiple follow-up CT scans were helpful for understanding the CT characteristics and evolution of the new lesions. Second, primary lesions in some patients were more extensive, which may cover or mix with new lesions during progression, thus making new lesions undetectable.

Table 3. Follow-up CT characteristics of COVID-19 patients with/without new lesions

\begin{tabular}{|c|c|c|c|}
\hline & $\begin{array}{l}\text { Patients with new } \\
\text { lesions }(n=42)\end{array}$ & $\begin{array}{l}\text { Patients without } \\
\text { new lesions } \\
(\mathrm{n}=14)\end{array}$ & $P$-value \\
\hline \multicolumn{4}{|l|}{ Follow-up time } \\
\hline Mean \pm SD (range) & $18 \pm 5(10-31)$ & $15 \pm 5(10-29)$ & $0.128^{\&}$ \\
\hline \multicolumn{4}{|l|}{$\begin{array}{l}\text { Interval between initial CT } \\
\text { and first negative nucleic } \\
\text { acid test }\end{array}$} \\
\hline Mean \pm SD (range) & $18 \pm 8(5-40)$ & $15 \pm 7(5-40)$ & $0.163^{\#}$ \\
\hline \multicolumn{4}{|l|}{ Changes of primary lesions } \\
\hline Absorption after progression & $38(90.5)$ & $2(14.3)$ & $0.000^{* *}$ \\
\hline Direct absorption & $4(9.5)$ & $12(85.7)$ & \\
\hline \multicolumn{4}{|l|}{ Changes of new lesions } \\
\hline Absorption after progression & $10(23.8)$ & - & \\
\hline Direct absorption & $32(76.2)$ & - & \\
\hline \multicolumn{4}{|l|}{$\begin{array}{l}\text { CT manifestations on latest } \\
\text { CT }\end{array}$} \\
\hline Pure GGOs & $9(21.4)$ & $9(64.3)$ & $0.006^{* *}$ \\
\hline $\begin{array}{l}\text { GGOs with fibrous stripes/ } \\
\text { consolidation }\end{array}$ & $33(78.6)$ & $5(35.7)$ & \\
\hline \multicolumn{4}{|c|}{$\begin{array}{l}\text { Note: Continuous data were presented as mean } \pm \text { standard deviation (minimum- } \\
\text { maximum), while the categorical data were presented as count (percentage of the } \\
\text { total); }\end{array}$} \\
\hline $\begin{array}{l}\text { * Pearson } \chi^{2} \text { test; }{ }^{* *} \text { Fisher exact } \\
\text { variance; }\end{array}$ & t; ; Wilcoxon rank s & sum test; \& Analysis & \\
\hline
\end{tabular}

\section{Conclusions}

Most patients with COVID-19 pneumonia would develop new pulmonary lesions during treatment, especially those with lymphopenia, elevated CRP, or primary lesion progression. These newly developed lesions usually manifest as multiple GGOs with or without consolidation, distribute around primary lesions or in the previously uninvolved lobes, and subsequently are absorbed directly. CT can be used along with nucleic acid tests to evaluate the outcomes of the disease and its infectivity.

\section{Abbreviations}

COVID-19: coronavirus disease 2019; SARS-CoV-2: severe acute respiratory syndrome coronavirus 2; GGOs: ground-glass opacities; PCR: polymerase chain reaction; CRP: C-reactive protein; $\mathrm{LDH}$ : lactate dehydrogenase; $\mathrm{PaO}_{2}$ : partial pressure of oxygen.

\section{Acknowledgments}

The work was funded by New Coronavirus Pneumonia Emergency Clinical Research project in Chongqing Medical University. 


\section{Authors' contributions}

Zhigang Chu designed the study, and participated in drafting the paper. Wangjia $\mathrm{Li}$ contributed in data analysis, and manuscript writing. Zhuoma Lv contributed in collecting data. Fajin Lv and Liangbo $\mathrm{Hu}$ reviewed critically preliminary results and commented on manuscript. Binjie $\mathrm{Fu}$ contributed in preparing figures and reviewed the statistical section.

\section{Competing Interests}

The authors have declared that no competing interest exists.

\section{References}

1. Li Q, Guan X, Wu P, et al. Early Transmission Dynamics in Wuhan, China, of Novel Coronavirus-Infected Pneumonia. N Engl J Med. 2020; 382: 1199-1207.

2. Zhu N, Zhang D, Wang W, et al. China Novel Coronavirus Investigating and Research Team. A Novel Coronavirus from Patients with Pneumonia in China, 2019. N Engl J Med. 2020; 382: 727-733.

3. Hui DS, I Azhar E, Madani TA, et al. The continuing 2019-nCoV epidemic threat of novel coronaviruses to global health-the latest 2019 novel coronavirus outbreak in Wuhan, China. Int J Infect Dis. 2020; 91: 264-266.

4. To KK, Tsang OT, Leung WS, et al. Temporal profiles of viral load in posterior oropharyngeal saliva samples and serum antibody responses during infection by SARS-CoV-2: an observational cohort study. Lancet Infect Dis. 2020; 20: 565-574.

5. Han Q, Lin Q, Ni Z, et al. Uncertainties about the transmission routes of 2019 novel coronavirus. Influenza Other Respir Viruses. 2020; 14: 470-471.

6. Sheridan C. Fast, portable tests come online to curb coronavirus pandemic. Nat Biotechnol. 2020; 38: 515-518.

7. Ai T, Yang Z, Hou H, et al. Correlation of Chest CT and RT-PCR Testing in Coronavirus Disease 2019 (COVID-19) in China: A Report of 1014 Cases. Radiology. 2020; 296: E32-E40.

8. Fang Y, Zhang H, Xie J, et al. Sensitivity of Chest CT for COVID-19: comparison to RT-PCR. Radiology. 2020; 296: E115-E117.

9. Chung M, Bernheim A, Mei X, et al. CT Imaging Features of 2019 Novel Coronavirus (2019-nCoV). Radiology. 2020; 295: 202-207.

10. Pan F, Ye T, Sun P, et al. Time Course of Lung Changes on Chest CT During Recovery From 2019 Novel Coronavirus (COVID-19) Pneumonia. Radiology. 2020; 295: 715-721.

11. Bernheim A, Mei X, Huang M, et al. Chest CT Findings in Coronavirus Disease-19 (COVID-19): Relationship to Duration of Infection. Radiology. 2020; 295: 200463.

12. Song F, Shi N, Shan F, et al. Emerging 2019 Novel Coronavirus (2019-nCoV) Pneumonia. Radiology. 2020; 295: 210-217.

13. Pan $\mathrm{Y}, \mathrm{Guan} \mathrm{H}$, Zhou $\mathrm{S}$, et al. Initial CT findings and temporal changes in patients with the novel coronavirus pneumonia (2019-nCoV): a study of 63 patients in Wuhan, China. Eur Radiol. 2020; 30: 3306-3309.

14. Wang D, Hu B, Hu C, et al. Clinical Characteristics of 138 Hospitalized Patients With 2019 Novel Coronavirus-Infected Pneumonia in Wuhan, China. JAMA. 2020; 323: 1061-1069.

15. Mo P, Xing Y, Xiao Y, et al. Clinical characteristics of refractory COVID-19 pneumonia in Wuhan, China. Clin Infect Dis 2020; [Epub ahead of print].

16. Tian S, Hu N, Lou J, et al. Characteristics of COVID-19 Infection in Beijing. J Infect. 2020; 80: 401-406.

17. Wu Z, McGoogan JM. Characteristics of and important lessons from the coronavirus disease 2019 (COVID-19) outbreak in China: summary of a report of 72314 cases from the Chinese Center for Disease Control and Prevention. JAMA 2020; [Epub ahead of print]

18. Chen $\mathrm{Y}, \mathrm{Li}$ L. SARS-CoV-2: virus dynamics and host response. Lancet Infect Dis. 2020; 20: 515-516.

19. Peiris JS, Chu CM, Cheng VC, et al. Clinical progression and viral load in a community outbreak of coronavirus-associated SARS pneumonia: a prospective study. Lancet. 2003; 361:1767-72.

20. Oh MD, Park WB, Choe PG, et al. Viral load kinetics of MERS coronavirus infection. N Engl J Med. 2016; 375: 1303-5.

21. Yoon SH, Lee KH, Kim JY, et al. Chest Radiographic and CT Findings of the 2019 Novel Coronavirus Disease (COVID-19): Analysis of Nine Patients Treated in Korea. Korean J Radiol. 2020; 21: 494-500.

22. Chung M, Bernheim A, Mei X et al. CT Imaging Features of 2019 Novel Coronavirus (2019-nCoV). Radiology. 2020; 295: 202-207.

23. Zu ZY, Jiang MD, Xu PP, et al. Coronavirus Disease 2019 (COVID-19): A Perspective from China. Radiology. 2020; 296: E15-E25. 